\title{
(2) OPEN ACCESS \\ Comprehensive Dutch market data analysis shows that e-liquids with nicotine salts have both higher nicotine and flavour concentrations than those with free-base nicotine
}

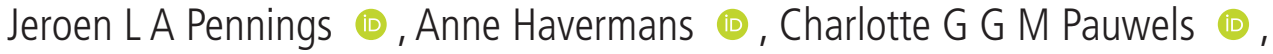 \\ Erna J Z Krüsemann, Wouter F Visser (1) , Reinskje Talhout
}

Centre for Health Protection National Institute for Public Health and the Environment (RIVM), Bilthoven, The Netherlands

\section{Correspondence to}

Dr Jeroen L A Pennings, National Institute for Public Health and the Environment, Bilthoven 3721 MA, The Netherlands; jeroen.pennings@rivm.nl

Received 31 July 2021 Accepted 28 December 2021
Check for updates

(C) Author(s) (or their employer(s)) 2022. Re-use permitted under CC BY-NC. No commercial re-use. See rights and permissions. Published by BMJ.

\begin{tabular}{|l|}
\hline To cite: Pennings JLA, \\
Havermans A, \\
Pauwels CGGM, et al. \\
Tob Control Epub ahead of \\
print: [please include Day \\
Month Year]. doi:10.1136/ \\
tobaccocontrol-2021-056952 \\
\hline
\end{tabular}

\section{ABSTRACT}

Objectives Recent years have seen an increase in e-liquids containing nicotine salts. Nicotine salts are less harsh and bitter than free-base nicotine and therefore can facilitate inhalation. Because inhalation-facilitating ingredients are banned in the European Union, we assessed the occurrence and characteristics of nicotine salt-containing e-liquids notified for the Netherlands. Methods We analysed data for 39030 products, submitted by manufacturers in the European Union Common Entry Gate system, as extracted on 30 June 2020.

Results Nicotine salts were present in $13 \%$ of e-liquids, especially in pod-related e-liquids (73\%) and e-liquids registered from 2018 onwards (over $25 \%$ ). We found six nicotine salt ingredients (NSIs): nicotine lactate, salicylate, benzoate, levulinate, ditartrate and malate. Nicotine salts also occurred as nicotine-organic acid ingredient combination (NAIC), like nicotine and benzoic acid. Nicotine concentrations were twofold higher in e-liquids with NSI (median $14 \mathrm{mg} / \mathrm{mL}$ ) and NAIC $(11 \mathrm{mg} /$ $\mathrm{mL}$ ) than for free-base nicotine $(6 \mathrm{mg} / \mathrm{mL})$. E-liquids with NSI contained a fourfold higher number (median $\mathrm{n}=17$ ) and concentration (median $31.0 \mathrm{mg} / \mathrm{mL}$ ) of flavour ingredients than e-liquids with free-base nicotine $(n=4$, $7.4 \mathrm{mg} / \mathrm{mL}$ ). In NAIC-containing e-liquids, these were threefold higher $(n=12,21.5 \mathrm{mg} / \mathrm{mL})$. E-liquids with nicotine salts were less often tobacco flavoured but more often had fruity or sweet flavours.

Conclusions A substantial and increasing share of eliquids in the Netherlands contains nicotine salts. Their characteristics can make such e-liquids more addictive and more attractive, especially to young and beginning users. Policymakers are advised to consider regulating products containing nicotine salts.

\section{INTRODUCTION}

Recent years have seen the introduction of new types of e-cigarettes, such as JUUL, that aerosolise e-liquid solutions from prefilled cartridges called 'pods'. In the USA, the introduction of JUUL e-cigarettes and similar devices coincided with a fast increase of e-cigarette use among young non-smokers. ${ }^{12}$ Common characteristics for JUUL and similar devices are an attractive design, high aerosol nicotine levels, e-liquids containing nicotine salts instead of free-based nicotine, and sweet and fruity flavours which are particularly appealing to young people. ${ }^{34}$

Alkaline free-base nicotine is bitter and a respiratory irritant. ${ }^{5}$ Additives that change nicotine from a free-base to a protonated chemical form can improve the sensory experience of vaping. ${ }^{6}$ In a recent randomised clinical trial, sensory reactions to puffs from e-cigarettes with nicotine salt (benzoic acid added) and nicotine free-base (no benzoic acid) formulations were assessed. Nicotine salt formulations resulted in statistically significant higher ratings of appeal, sweetness and smoothness, and lower ratings of bitterness and harshness. These effects were more pronounced among never-smokers unaccustomed to inhaling free-base nicotine than in ever-smokers. ${ }^{6}$ This study showed that formulations containing nicotine salt rather than free-base can enhance the appeal and sensory experience of vaping and advised that they merit consideration in e-cigarette regulation. Additionally, formulations that are less harsh are more likely to be inhaled into the lungs rather than kept in the oral cavity. ${ }^{7}$ Increased delivery of nicotine to the lungs will lead to greater absorption into the bloodstream. Hence, the use of nicotine salts can make inhalation of nicotine more palatable ${ }^{68}$ and increase nicotine blood concentrations. ${ }^{7}$ However, additives that facilitate inhalation or nicotine uptake are forbidden according to the European Tobacco Products Directive under articles 7 (6d) and $20(3 \mathrm{c}){ }^{9}$

While JUUL is not available on the Dutch market, many e-liquids with nicotine salt ingredients (NSIs) or nicotine-organic acid ingredient combination (NAIC) have been notified to the Dutch section of the European Union Common Entry Gate (EUCEG).$^{10}$ EU-CEG is a non-public database in which manufacturers and importers are legally obliged to provide information about the composition and other properties of tobacco and e-cigarette products they market in each EU country. ${ }^{9}$ Product data need to be notified as least 6 months before a product enters a national market, updated if there are changes to a product and get labelled as inactive if a product is no longer for sale. EU-CEG data then become accessible to the relevant competent national authorities. ${ }^{9}$ We used these EU-CEG data to create an overview of these products and their characteristics. This will provide relevant insights for Dutch and international policymaking.

\section{METHODS}

We used e-cigarette product data as available as active products in the Dutch section of the EU-CEG system on 30 June 2020. Product data were exported and further statistical analyses were carried out in 
Table 1 Number of products by EU-CEG product type and nicotine class

\begin{tabular}{|c|c|c|c|c|c|}
\hline Product type & Absent & Free-base & NAIC & NSI & Total \\
\hline 1. Electronic cigarette-disposable & 70 & 630 & 194 & 155 & 1049 \\
\hline 2. Electronic cigarette-rechargeable & 4 & 37 & 7 & 2 & 50 \\
\hline 3. Electronic cigarette-rechargeable, device only & 979 & 4 & 6 & 0 & 989 \\
\hline 4. Electronic cigarette-refillable & 6 & 26 & 2 & 0 & 34 \\
\hline 5. Electronic cigarette-refillable, device only & 2996 & 6 & 0 & 1 & 3003 \\
\hline 6. Kit—-pack with more than one device and/or refill & 2099 & 46 & 40 & 3 & 2188 \\
\hline 9. Other & 695 & 291 & 0 & 0 & 986 \\
\hline Total & 13060 & 22082 & 3026 & 862 & 39030 \\
\hline
\end{tabular}

Nicotine classes: absent, nicotine absent; free-base, free-base nicotine.

EU-CEG, European Union Common Entry Gate; NAIC, nicotine-organic acid ingredient combination; NSI, nicotine salt ingredient.

R (V.4.0.2) and Microsoft Excel. Overall, we obtained data for 39030 e-cigarette products that were notified in the period from July 2016 up to and including June 2020.

We searched for nicotine or its salts in the ingredient data fields 'ingredient name' and 'CAS-number' as provided by the submitter. A CAS-number is a unique numerical identifier assigned by the Chemical Abstracts Service to every chemical substance described in the open scientific literature, the numerical code does not contain structural information by itself. Our search terms for ingredient name were "nicot"”, "nikot" and "pyrid"”, followed by manual curation. Our search terms for CAS-number were the known CAS-numbers for nicotine and additionally the CAS-numbers for nicotine salts identified by ingredient name searches. Additionally, we identified which products contained any of the organic acids that are needed to form the nicotine salts found in our dataset.

Products for each EU-CEG product type were assigned to one of the following nicotine classes, based on the type of ingredients as declared in EU-CEG: no nicotine; free-base nicotine; NAIC, that is, nicotine combined with one or more of the organic acids; NSI, that is, products with nicotine salts (including products that contain both NSI and free-base nicotine with or without organic acid). Most products containing nicotine or nicotine salts belonged to disposable (EU-CEG product type 1, total $\mathrm{n}=1049$ ) or refill (product type 7 , total $\mathrm{n}=28585$ ) product types (table 1). Rechargeable (product type 2 , total $n=50$ ) and refillable (product type 4 , total $n=34$ ) products were excluded from further analyses as the number of products with nicotine salts in these categories ( $n=9$ and $n=2$, respectively) was too low for meaningful analyses (table 1). Other product types such as 'devices only' or 'individual part' were also excluded as not being relevant.

We also performed nicotine type classification on the subset of pod e-liquids. Because EU-CEG does not have a mandatory data field to indicate whether a product is pod related, we based our analysis on e-liquids from known pod brands or e-liquids for which the EU-CEG data contained a description indicating that the product was a pod e-liquid. Additionally, we performed nicotine type classification on e-liquids by their year of EU-CEG submission, to see if NSI and NAIC are increasingly notified.

For the four nicotine classes in the set of disposable and refill e-liquids, we used the EU-CEG data to determine the median nicotine concentration and the IQR, as well as the percentage of liquids above the legal limit of $20 \mathrm{mg} / \mathrm{mL}$. We also determined the median and IQR of the acid/nicotine ratio, which is the molar ratio between organic acid and nicotine, to get an indication of the protons available for protonating nicotine. Additionally, we determined the median and IQR number of ingredients and flavourings per product, as well as the median and IQR number concentration of flavourings.

For flavour classification of e-liquids, product descriptions available in EU-CEG and on the internet were used to assign an e-liquid to a flavour wheel category, ${ }^{11}$ using the procedure described by Havermans et al. ${ }^{12}$

\section{RESULTS}

\section{Nicotine salts}

We found six specific NSI declared in the EU-CEG dataset (nicotine benzoate, ditartrate, lactate, levulinate, malate and salicylate), as well as some ingredient names that we grouped as 'unspecified NSI'. This latter group had an ingredient name indicating a nicotine salt (ie, 'nicotine salt', 'salt of nicotine' or 'sel de nicotine'), but lacked additional specification such as a CAS-number. Overall ( $n=39030$ e-cigarette products), nicotine lactate was the most frequently used salt $(n=303)$, followed by nicotine salicylate $(n=218)$, nicotine benzoate $(n=188)$, nicotine levulinate $(n=66)$, unspecified NSI $(n=63)$, nicotine ditartrate $(n=24)$ and nicotine malate $(n=6)$. In addition to these NSIs, we found products that contain nicotine as well as (an) organic acid(s) which together form one of the above NSIs. For these NAICs, the number of products was higher but the relative order was similar: the most frequent resulting salt was nicotine lactate $(n=1057)$, followed by nicotine benzoate $(n=1020)$, nicotine salicylate $(n=609)$, nicotine malate $(n=624)$, nicotine levulinate $(n=217)$ and nicotine ditartrate $(n=90)$.

\section{Products}

The majority (98\%) of products containing nicotine or nicotine salts belonged to disposables or refills (table 1). In disposables $(n=1049), 155(14.8 \%)$ contained NSI and 194 (18.5\%) NAIC. In refills $(\mathrm{n}=28585), 701(2.4 \%)$ contained NSI and 2767 (9.7\%) NAIC (table 1). This indicates that disposables more often contained nicotine salts than refills. When combined, $12.9 \%$ of disposable or refill e-liquids contained nicotine salts.

Of the pod-like (disposable or refill) e-liquids ( $n=570), 28.8 \%$ contained an NSI and 44.6\% an NAIC. For non-pod (disposable or refill) e-liquids $(n=29064)$, these values were $2.4 \%$ and $9.3 \%$, respectively. Therefore, pods contributed substantially, although not exclusively, to the percentage of e-liquids with nicotine salts in the overall set of e-liquids.

A breakdown of nicotine class by year of EU-CEG submissions showed that the percentage of notified e-liquids with NSI was $0.1 \%$ for those submitted in 2016 (total $n=14134$ ), 
Table 2 Characteristics of disposable and refill e-liquids by nicotine class

\begin{tabular}{lllll}
\hline Product type & $\begin{array}{l}\text { Absent } \\
(\mathbf{n}=4189)\end{array}$ & $\begin{array}{l}\text { Free-base } \\
(\mathbf{n}=21628)\end{array}$ & $\begin{array}{l}\text { NAIC } \\
(\mathbf{n}=2961)\end{array}$ & $\begin{array}{l}\text { NSI } \\
(\mathbf{n}=856)\end{array}$ \\
\hline Nicotine (or nicotine equivalent) concentration $(\mathrm{mg} / \mathrm{mL})$ & 0 & $6(2-12)$ & $11(6-20)$ & $14(7-25)$ \\
Number of ingredients & $6(2-13)$ & $8(5-15)$ & $16(9-34)$ & $20(11-30)$ \\
Number of flavour ingredients & $3(0-9)$ & $4(1-11)$ & $12(5-30)$ & $17(7-27)$ \\
Flavour ingredients concentration $(\mathrm{mg} / \mathrm{mL})$ & $1.8(0-25.7)$ & $7.4(0.3-30.0)$ & $21.5(10.7-47.1)$ & $31.0(14.9-69.6)$ \\
\hline
\end{tabular}

Values are median (IQR) values for the parameters mentioned. Nicotine classes: absent, nicotine absent; free-base, free-base nicotine.

NAIC, nicotine-organic acid ingredient combination; NSI, nicotine salt ingredient.

1.4\% for $2017(n=4699)$, vs $8.8 \%$ for $2018(n=2901), 6.4 \%$ for $2019(n=5905)$ and $9.4 \%$ for $2020(n=1545)$. For NAIC, these percentages were $4.4 \%$ for $2016,5.0 \%$ for 2017 , vs $21.2 \%$ for 2018, 19.2\% for 2019 and $22.0 \%$ for 2020. This indicates that nicotine salts are used more frequently in the e-liquids from 2018 onwards.

\section{Concentrations}

For each nicotine class, the median concentration of nicotine or its salts was determined. For e-liquids with free-base nicotine, this was $6 \mathrm{mg} / \mathrm{mL}$ (IQR 2-12 $\mathrm{mg} / \mathrm{mL}$ ), for NAIC $11 \mathrm{mg} / \mathrm{mL}$ (IQR $6-20 \mathrm{mg} / \mathrm{mL}$ ) and for NSI $14 \mathrm{mg} / \mathrm{mL}$ (IQR $7-25 \mathrm{mg} / \mathrm{mL}$ ) (all expressed as free-base nicotine weight equivalent) (table 2). Remarkably, according to EU-CEG data, 36\% of the e-liquids with NSI had nicotine concentrations higher than the legal limit ${ }^{9}$ of $20 \mathrm{mg} / \mathrm{mL}$ (expressed as free-base equivalent), whereas this was only $2 \%$ for e-liquids with free-base nicotine and $4 \%$ for NAIC. In most cases, such e-liquids had concentrations between 20 and $30 \mathrm{mg} / \mathrm{mL}$, as the percentage of products exceeding $30 \mathrm{mg} / \mathrm{mL}$ was $16 \%$ for e-liquids with NSI, $1 \%$ for e-liquids with free-base nicotine and $1 \%$ for NAIC.

To assess to what extent a combined presence of nicotine and an organic acid is indeed equivalent to the presence of an NSI, we determined acid/nicotine ratios. The overall median acidnicotine ratio was $72 \%$ of an equimolar concentration (IQR 25\%-130\%), which would be sufficient for about two-thirds of the nicotine to become protonated. Median acid-nicotine ratios for the most frequently used acids were similar (lactic acid 71\% (IQR 17\%-122\%), benzoic acid 78\% (IQR 38\%-103\%), salicylic acid 97\% (IQR 56\%-108\%), malic acid 40\% (IQR 26\%-99\%)). These ratios suggest that organic acids are used to protonate nicotine and thus obtain an NSI. This is corroborated by the fact that overall $12 \%$ of e-liquids with free-base nicotine contained one of these organic acids (based on the ratio of NAIC over the combined free-base nicotine and NAIC groups), vs only $4 \%$ of the e-liquids without nicotine. However, several of these acids have a low-strength or medium-strength odour (benzoic acid balsamic, salicylic acid phenolic/nutty, levulinic acid caramellic) and for some e-liquids they are declared in EU-CEG as flavouring ingredients, so an (additional) function as flavouring ingredient cannot be ruled out. More specifically, for $71 \%$ of the NAIC e-liquids, the acid is notified as 'flavour or flavour enhancer', for $24 \%$ as ' $\mathrm{pH}$ modifier' and for $4 \%$ as 'other function'.

\section{Flavours}

We determined the median number of total ingredients, as well as of ingredients listed as 'flavour or flavour enhancer', in the various nicotine type groups. For e-liquids without nicotine, the median number of ingredients was 6 (IQR 2-13), for e-liquids with free-base nicotine 8 (IQR 5-15), for e-liquids with NAIC 16 (IQR 9-34) and for e-liquids with NSI 20 (IQR 11-30) (table 2). For flavour ingredients, these values were 3 (IQR 0-9), 4 (IQR 1-11), 12 (IQR 5-30) and 17 (IQR 7-27), respectively (table 2). So, e-liquids with NSI, and to a lesser extent those with NAIC, contained more ingredients than e-liquids with freebase nicotine or no nicotine, which can mainly be attributed to an increased use of flavour ingredients. Other ingredient functions (EU-CEG defines 26 functions) did not show clear differences between nicotine classes. A similar increase was observed in the overall concentration of flavour ingredients. For e-liquids without nicotine, the median concentration was $1.8 \mathrm{mg} / \mathrm{mL}$ (IQR 0-25.7), for free-base nicotine 7.4 (IQR 0.3-30.0), for NAIC 21.5 (IQR 10.7-47.1) and for NSI 31.0 (IQR 14.9-69.6) mg/ $\mathrm{mL}$ (table 2).

We also classified e-liquids by the categories of the e-liquid flavour wheel. ${ }^{11}$ This revealed a difference in the distribution across flavour categories (figure 1). Most notably, e-liquids with NSI or NAIC were both less often tobacco flavoured, and more often had fruity flavours and sweet flavours such as dessert and candy, compared with e-liquids with free-base nicotine or without nicotine.

\section{DISCUSSION}

Our findings strengthen several of the concerns regarding e-liquids with nicotine salts. E-liquids with nicotine salts were found to contain nicotine concentrations that were about twice that of e-liquids with free-base nicotine. This supports the hypothesis that nicotine salts are intended to reduce the bitterness and harshness of nicotine, as these would otherwise become too pronounced at higher concentrations. ${ }^{4} 6$ Higher nicotine concentrations, combined with reduced throat irritation, may make nicotine salt e-liquids more attractive and addictive than e-liquids with free-base nicotine. Moreover, both their number of flavour ingredients and their total concentration of flavour ingredients are three to four times that of e-liquids with freebase nicotine. E-liquids with nicotine salts also more often had fruity or sweet flavours not found in traditional tobacco products. Such non-traditionally flavoured e-liquids are used at e-cigarette initiation by the majority of youth because these flavours enhance the appeal of e-cigarettes by masking the aversive taste of nicotine. ${ }^{13}$ Moreover, sweet flavours are preferred by adolescents and-less exclusively-also by young adults, as well as adults. ${ }^{14-16}$ It can be remarked here that the overall implications of nicotine salts may not be fully comparable between, for example, young non-smokers who experiment with e-cigarette use versus adult smokers who use e-cigarettes to attempt quitting smoking tobacco products. ${ }^{7}$ However, taken together, the combined increase in addictiveness and attractiveness may warrant specific regulations for nicotine salt e-liquids.

Although our study focused on the Dutch market, and thus may not be fully representative for other international markets, many e-liquids notified for the Netherlands are also notified for other EU countries and (also) have product description data 


\section{Flavor category}

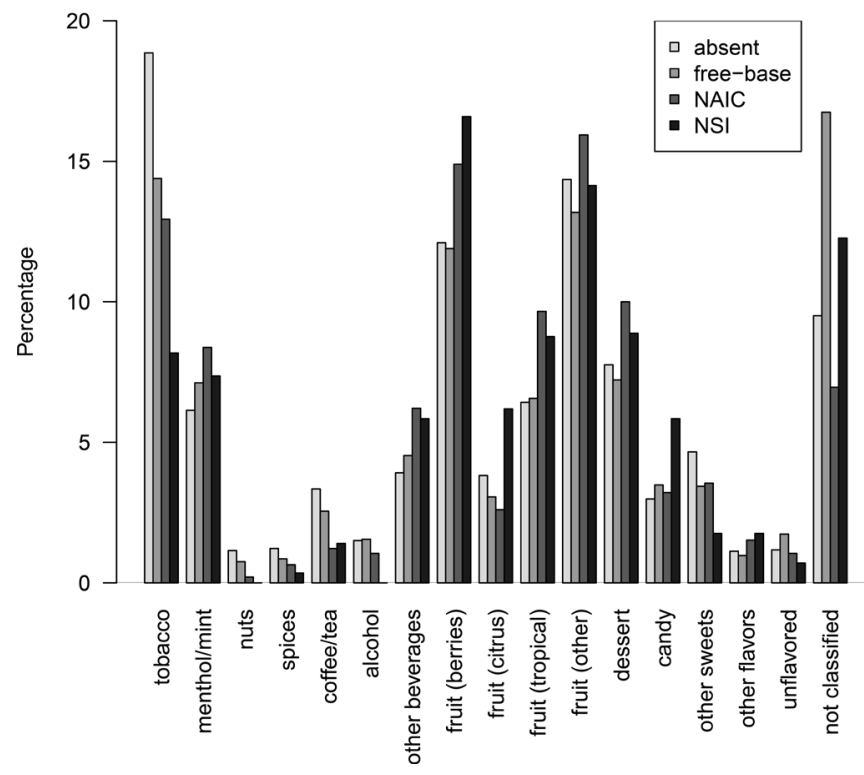

Figure 1 Percentage distribution of flavour wheel categories by nicotine class: absent, nicotine absent; free-base, free-base nicotine. NAIC, nicotine-organic acid ingredient combination; NSI, nicotine salt ingredient.

in languages other than Dutch, so many of our findings can be extended at least to other European markets.

Our analyses are based on data that are entered into EU-CEG by e-cigarette manufacturers or importers. It is not always possible or feasible to ensure the data are correct. Occasionally, we found entries for which we suspected that data may have been entered incorrectly into the database. To avoid such entries influencing the results, results were reported as robust measures such as a median instead of an average. Also, EU-CEG does not declare whether an e-liquid concerns a pod, so as this had to be inferred from other data fields, we may have underestimated the number of pod products. Given these analytical limitations, some caution may need to be kept in mind when interpreting the data.

\section{What this paper adds}

What is already known on this subject

- Nicotine salts are less harsh and bitter than free-base nicotine, can improve the sensory experience of vaping and may facilitate inhalation. In recent years, the introduction of nicotine salt-based types of e-cigarettes coincided with a fast increase of e-cigarette use among young non-smokers.

What important gaps in knowledge exist on this topic

- Most studies on nicotine salt-containing e-liquids are based on the US market or focus on specific e-cigarette brands.

\section{What this study adds}

-We analysed the e-liquid composition data for the complete set of e-liquids notified as active on the Dutch market in June 2020. We found that $13 \%$ of the e-liquids contain nicotine salts and these liquids have characteristics that point at an increased addictiveness and attractiveness. Our results can be used for assessing trends in e-liquids composition and developing regulation of nicotine salt e-liquids.
Two of our findings regarding e-liquids with nicotine salts, namely the larger number of flavouring ingredients and the larger share of fruity and sweet flavours, may be connected to each other. Recent studies by Krüsemann et al also found that e-liquids with fruity or sweet flavours have a larger number of ingredients than, for example, tobacco flavour. ${ }^{17} 18$

Most nicotine salt e-liquids did not have the actual salt listed as an ingredient, but rather had nicotine and an organic acid listed as separate ingredients. In such cases, we only considered this as a nicotine salt if that particular acid would result in one of the NSIs found in our dataset, because for these acids we had evidence that they were used as such. However, as there were several other acids declared as ingredients in EU-CEG, including several flavouring ingredients such as butyric acid or phenylacetic acid, that can all contribute to nicotine protonation, we may have missed some NAICs and thus underestimated their occurrence.

Although e-liquids with NSI as well as NAIC have higher nicotine concentrations and numbers and concentration of flavour ingredients, compared with e-liquids with free-base nicotine (or without nicotine), this difference was less pronounced in e-liquids with NAIC than in those with NSI. Nevertheless, the large number of e-liquids with NAIC, the increase herein during recent years, as well as the larger share of fruity and sweet flavours, provide reason to extend the concerns regarding nicotine salts also to those with NAIC.

Overall, policymakers are advised to consider regulation of nicotine salt e-liquids, both NSIs and NAICs, given their high sensory appeal, especially among never-smokers, ${ }^{6}$ flavours attractive to youth and their high nicotine content. More specifically, policymakers could consider limiting the use of nicotine salts, setting (quantitative) limits to acid ingredients, limiting the extent of nicotine protonation or restricting the $\mathrm{pH}$ range of e-liquids.

Contributors RT conceived the research idea, secured funding and acts as guarantor for the study. JLAP carried out the product data analyses and drafted the manuscript. All authors contributed to the research plan and helped revise the manuscript. All authors approved the final version for publication.

Funding The authors have not declared a specific grant for this research from any funding agency in the public, commercial or not-for-profit sectors.

Competing interests None declared.

Patient consent for publication Not required.

Ethics approval This study does not involve human participants.

Provenance and peer review Not commissioned; externally peer reviewed.

Data availability statement Data are available upon reasonable request.

Open access This is an open access article distributed in accordance with the Creative Commons Attribution Non Commercial (CC BY-NC 4.0) license, which permits others to distribute, remix, adapt, build upon this work non-commercially, and license their derivative works on different terms, provided the original work is properly cited, appropriate credit is given, any changes made indicated, and the use is non-commercial. See: http://creativecommons.org/licenses/by-nc/4.0/.

\section{ORCID iDs}

Jeroen L A Pennings http://orcid.org/0000-0002-9188-6358

Anne Havermans http://orcid.org/0000-0003-2874-6560

Charlotte G G M Pauwels http://orcid.org/0000-0001-6727-0804

Wouter F Visser http://orcid.org/0000-0003-1129-262X

\section{REFERENCES}

1 Kavuluru R, Han S, Hahn EJ. On the popularity of the USB flash drive-shaped electronic cigarette Juul. Tob Contro/ 2019;28:110-2.

2 Ramamurthi D, Chau C, Jackler RK. JUUL and other stealth vaporisers: hiding the habit from parents and teachers. Tob Control 2019;28:610-6. doi:10.1136/ tobaccocontrol-2018-054455 
3 Jackler RK, Ramamurthi D. Nicotine arms race: JUUL and the high-nicotine product market. Tob Control 2019;28:623-8.

4 Barrington-Trimis JL, Leventhal AM. Adolescents' Use of "Pod Mod" E-Cigarettes Urgent Concerns. N Engl J Med 2018;379:1099-102.

5 DeVito EE, Krishnan-Sarin S. E-Cigarettes: impact of E-Liquid components and device characteristics on nicotine exposure. Curr Neuropharmacol 2018;16:438-59.

6 Leventhal AM, Madden DR, Peraza N, et al. Effect of exposure to e-cigarettes with salt vs Free-Base nicotine on the appeal and sensory experience of Vaping: a randomized clinical trial. JAMA Netw Open 2021:4:e2032757.

7 O'Connell G, Pritchard JD, Prue C, et al. A randomised, open-label, cross-over clinical study to evaluate the pharmacokinetic profiles of cigarettes and ecigarettes with nicotine salt formulations in US adult smokers. Intern Emerg Med 2019;14:853-61.

8 Duell AK, Pankow JF, Peyton DH. Nicotine in tobacco product aerosols: 'It's déjà vu all over again'. Tob Control 2020;29:656-62.

9 European Union. Tobacco Products Directive 2014/40/EU of the European Parliament and of the Council, 2014. Available: https:/ec.europa.eu/health/sites/health/files/ tobacco/docs/dir_201440_en.pdf

10 European Commission. EU common entry gate (EU-CEG), 2016. Available: https://ec. europa.eu/health/euceg/
11 Krüsemann EJZ, Boesveldt S, de Graaf K, et al. An E-Liquid flavor wheel: a shared vocabulary based on systematically reviewing E-Liquid flavor classifications in literature. Nicotine Tob Res 2019;21:1310-9.

12 Havermans A, Krüsemann EJZ, Pennings J, et al. Nearly 20000 e-liquids and 250 unique flavour descriptions: an overview of the Dutch market based on information from manufacturers. Tob Control 2021;30:57-62.

13 Goldenson NI, Leventhal AM, Simpson KA, et al. A review of the use and appeal of flavored electronic cigarettes. Curr Addict Rep 2019;6:98-113.

14 Krüsemann EJZ, van Tiel L, Pennings JLA, et al. Both nonsmoking youth and smoking adults like sweet and Minty E-liquid flavors more than tobacco flavor. Chem Senses 2021;46:1. doi:10.1093/chemse/bjab009

15 Zare S, Nemati M, Zheng Y. A systematic review of consumer preference for e-cigarette attributes: flavor, nicotine strength, and type. PLoS One 2018;13:e0194145.

16 Meernik C, Baker HM, Kowitt SD, et al. Impact of non-menthol flavours in e-cigarettes on perceptions and use: an updated systematic review. BMJ Open 2019;9:e031598.

17 Krüsemann EJZ, Pennings JLA, Cremers JWJM, et al. GC-MS analysis of e-cigarette refill solutions: a comparison of flavoring composition between flavor categories. J Pharm Biomed Anal 2020;188:113364.

18 Krüsemann EJZ, Havermans A, Pennings JLA, et al. Comprehensive overview of common e-liquid ingredients and how they can be used to predict an e-liquid's flavour category. Tob Control 2021;30:185-91. 